\title{
Solvability of Linear Differential Systems with Small Exponents in the Liouvillian Sense
}

\author{
R. R. Gontsov ${ }^{1}$ - I. V. Vyugin ${ }^{1,2}$
}

Received: 25 November 2014 / Revised: 20 August 2015 / Accepted: 11 November 2015 /

Published online: 26 November 2015

(C) Institute for Mathematical Sciences (IMS), Stony Brook University, NY 2015

\begin{abstract}
The paper is devoted to solvability of linear differential systems by quadratures, one of the classical problems of differential Galois theory. As known, solvability of a system depends entirely on properties of its differential Galois group. However, detecting solvability or non-solvability of a given system is a difficult problem, because the dependence of its differential Galois group on the coefficients of the system remains unknown. We consider systems with regular singular points as well as those with nonresonant irregular ones, whose exponents (respectively, so-called formal exponents in the irregular case) are sufficiently small. It turns out that for systems satisfying such restrictions criteria of solvability can be given in terms of the coefficient matrix.
\end{abstract}

Keywords Picard-Vessiot extension · Differential Galois group · Liouvillian solution $\cdot$ Stokes matrices

Mathematics Subject Classification $\quad 34 \mathrm{M} 03 \cdot 34 \mathrm{M} 15 \cdot 34 \mathrm{M} 35 \cdot 12 \mathrm{H} 05$

$\triangle$ I. V. Vyugin

vyugin@gmail.com

R. R. Gontsov

gontsovrr@gmail.com

1 Insitute for Information Transmission Problems RAS, Bolshoy Karetny per. 19, Moscow 127994, Russia

2 National Research University Higher School of Economics, Vavilova str. 7, Moscow 117312, Russia 


\section{Introduction}

Consider on the Riemann sphere $\overline{\mathbb{C}}$ a linear differential system

$$
\frac{d y}{d z}=B(z) y, \quad y(z) \in \mathbb{C}^{p},
$$

of $p$ equations with a meromorphic coefficient matrix $B$ (whose entries are thus rational functions) and singularities at some points $a_{1}, \ldots, a_{n}$. Solvability of linear differential equations and systems in the Liouvillian sense (in other words, by quadratures) is a classical problem of differential Galois theory developed by Picard and Vessiot at the beginning of the twentieth century. In analogy to Galois theory of algebraic equations, they connected to the system a group called the differential Galois group and showed that solvability of the system by quadratures depends entirely on properties of its differential Galois group. Later, in the middle of the century, Kolchin completed this theory by considering other types of solvability and their dependence on properties of the differential Galois group.

However, the Picard-Vessiot-Kolchin theory reveals the cause of solvability or non-solvability of linear differential equations by quadratures rather than answers this question addressed to a specific equation, since one does not know how the differential Galois group of an equation depends on its coefficients. In our paper, we are interested in the cases when the answer to the question of solvability of the system (1) by quadratures can be given in terms of its coefficient matrix $B$. For example, in the case of a Fuchsian system

$$
\frac{d y}{d z}=\left(\sum_{i=1}^{n} \frac{B_{i}}{z-a_{i}}\right) y, \quad B_{i} \in \operatorname{Mat}(p, \mathbb{C}),
$$

with sufficiently small entries of the matrices $B_{i}$, Ilyashenko and Khovanskii (1974) (see also Khovanskii 2008, Ch. 6, §2.3) obtained an explicit criterion of solvability. Namely, the following statement holds:

There exists an $\varepsilon=\varepsilon(n, p)>0$ such that a condition of solvability by quadratures for the Fuchsian system (2) with $\left\|B_{i}\right\|<\varepsilon$ takes an explicit form: the system is solvable by quadratures if and only if all the matrices $B_{i}$ are triangular (in some common basis).

Using results of Kolchin, these authors also obtained criteria for other types of solvability of the Fuchsian system (2) with small residue matrices $B_{i}$ in terms of these matrices. Moreover, a topological version of Galois theory developed by Khovanskii at the beginning of 1970's allowed him to obtain stronger results concerning nonsolvability of Fuchsian systems.

Our paper may be considered as an attempt to generalize results of Ilyashenko and Khovanskii to the case of non-Fuchsian systems. It is organized as follows. In the next two sections we recall basic notions of differential Galois theory and of the local theory of linear differential systems, which are used in the paper.

In Sect. 4, we describe a weak version of Ramis's theorem (due to Ilyashenko and Khovanskii) concerning the description of the local differential Galois group at a singular point of a system in terms of local meromorphic invariants of the latter. 
This theorem is essentially used to prove statements on solvability of non-Fuchsian systems by quadratures. In this section we also recall the notion of exponential torus, a subgroup of the local differential Galois group, which also appears in further proofs.

Section 5 is devoted to an approach that uses holomorphic vector bundles with meromorphic connections on the Riemann sphere in a context of the analytic theory of linear differential equations. This approach was developed and successfully applied by Bolibrukh to solving inverse monodromy problems of this theory, in particular, Hilbert's 21st (the Riemann-Hilbert) problem. Here we also use this approach, combining it with techniques from differential Galois theory, to obtain statements concerning solvability of linear differential systems by quadratures.

Section 6 is about solvability of Fuchsian systems (more generally, systems with regular singular points) by quadratures. In particular, we refine here the IlyashenkoKhovanskii criterion in such a way that it is sufficient that the eigenvalues of the residue matrices $B_{i}$ be small (the estimate is given) rather than the matrices themselves. This refinement is naturally extended to other types of solvability, as well as to strong non-solvability of Fuchsian systems.

In Sect. 7, we propose a generalization of the criterion of solvability by quadratures to the case of essentialy non-Fuchsian systems (systems with non-resonant irregular singular points) with small formal exponents, and also discuss other types of solvability, including local solvability by quadratures over the field of meromorphic germs at an irregular singular point.

\section{Solvability by Quadratures and the Differential Galois Group}

In this section, we recall the definitions of some basic notions of differential Galois theory. Besides solvability of a linear differential system by quadratures, we consider other types of solvability and explain how they depend on properties of the differential Galois group of the system.

A Picard-Vessiot extension of the field $\mathbb{C}(z)$ of rational functions corresponding to the system (1) is a differential field $F=\mathbb{C}(z)\langle Y\rangle$ generated as a field extension of $\mathbb{C}(z)$ by all the entries of a fundamental matrix $Y(z)$ of the system (1). Let us specify that such a matrix is taken (by Cauchy's theorem) in the field of germs of meromorphic functions at a non-singular point $z_{0}$ of the system. One says that the system (1) is solvable by quadratures, if there is a fundamental matrix $Y$, whose entries are expressed in elementary or algebraic functions and their integrals, or, more formally, if the field $F$ is contained in some differential field extension of $\mathbb{C}(z)$ generated by algebraic functions, integrals and exponentials of integrals:

$$
\mathbb{C}(z)=F_{1} \subset \cdots \subset F_{m}, \quad F \subseteq F_{m},
$$

where each $F_{i+1}=F_{i}\left\langle x_{i}\right\rangle$ is a field extension of $F_{i}$ by an element $x_{i}$, which is either:

- algebraic over $F_{i}$, or

- an integral (that is, an element whose derivative belongs to $F_{i}$ ), or

- an exponential of an integral (that is, an element whose logarithmic derivative belongs to $F_{i}$ ). 
Such an extension $\mathbb{C}(z) \subseteq F_{m}$ is called Liouvillian; thus solvability by quadratures means that the Picard-Vessiot extension $F$ is contained in some Liouvillian extension of the field of rational functions.

In analogy to classical Galois theory, solvability or non-solvability of a linear differential system by quadratures is related to properties of its differential Galois group. The differential Galois group $\mathbf{G}=\mathrm{Gal}(F / \mathbb{C}(z))$ of the system (1) (of a Picard-Vessiot extension $\mathbb{C}(z) \subseteq F$ ) is the group of differential automorphisms of the field $F$ (i.e., automorphisms commuting with differentiation) that preserve the elements of the field $\mathbb{C}(z)$ :

$$
\mathbf{G}=\left\{\sigma: F \rightarrow F \mid \sigma \circ \frac{d}{d z}=\frac{d}{d z} \circ \sigma, \sigma(f)=f \quad \forall f \in \mathbb{C}(z)\right\}
$$

(The differential Galois group can be defined by any Picard-Vessiot extension, since these are all isomorphic as differential fields.) As follows from the definition, the image $\sigma(Y)$ of the fundamental matrix $Y$ of the system (1) under any element $\sigma$ of the Galois group is a fundamental matrix of this system again. Hence, $\sigma(Y)=Y C, C \in \mathrm{GL}(p, \mathbb{C})$. As every element of the differential Galois group is determined uniquely by its action on a fundamental matrix of the system, the group $\mathbf{G}$ can be regarded as a subgroup of the matrix group $\operatorname{GL}(p, \mathbb{C})$ for any such $Y$. Moreover, this subgroup $\mathbf{G} \subseteq \mathrm{GL}(p, \mathbb{C})$ is algebraic, i.e., closed in the Zariski topology of the space $\mathrm{GL}(p, \mathbb{C})$ (the topology, whose closed sets are those determined by systems of polynomial equations), see (Kaplansky 1957, Th. 5.5). A very good reference for the basics of differential Galois theory, especially the algebraic geometric aspects, is the book by Crespo and Hajto (2011).

The differential Galois group $\mathbf{G}$ is a union of a finite number of disjoint connected sets that are open and closed simultaneously (in the Zariski topology), and the set containing the identity matrix is called the identity component. The identity component $\mathbf{G}^{0} \subseteq \mathbf{G}$ is a normal subgroup of finite index (Kaplansky 1957, Lemma 4.5). According to the Picard-Vessiot theorem, solvability of the system (1) by quadratures is equivalent to solvability of the subgroup $\mathbf{G}^{0}$ (see Kaplansky 1957, Th. 5.12; Khovanskii 2008, Ch. 3, Th. 5.1). Recall that a group $H$ is said to be solvable, if there exist intermediate subgroups $\{e\}=H_{0} \subset H_{1} \subset \cdots \subset H_{m}=H$, such that, for every $i=1, \ldots, m$, $H_{i-1}$ is normal in $H_{i}$ and the factor group $H_{i} / H_{i-1}$ is Abelian.

Alongside the differential Galois group, one considers the monodromy group $\mathbf{M}$ of the system (1) generated by the monodromy matrices $M_{1}, \ldots, M_{n}$ corresponding to analytic continuation of the fundamental matrix $Y$ around the singular points $a_{1}, \ldots, a_{n}$. Each $M_{i}$ is defined as follows: since the operation of analytic continuation commutes with differentiation, the matrix $Y$ considered in a neighbourhood of a non-singular point $z_{0}$ goes to another fundamental matrix, $Y M_{i}$, under an analytic continuation along a simple loop $\gamma_{i}$ encircling a point $a_{i}$ and no other $a_{j}$. As analytic continuation also preserves elements of the field $\mathbb{C}(z)$ (since they are single-valued functions), one has $\mathbf{M} \subseteq \mathbf{G}$. Furthermore, the differential Galois group of a Fuchsian system coincides with the closure of its monodromy group in the Zariski topology (see Khovanskii 2008, Ch. 6, Cor. 1.3). Hence, a Fuchsian system is solvable by 
quadratures if and only if the identity component $\mathbf{M} \cap \mathbf{G}^{0}$ of its monodromy group is solvable.

Now we will discuss other types of solvability. These are defined in analogy to solvability by quadratures, and we leave formal definitions to the reader. Kolchin (1948) gave criteria for a linear differential system to be solvable with respect to each of these types in terms of its differential Galois group. Further we will need his results only for a system, whose differential Galois group is triangular, meaning that all matrices of the group are triangular in some common basis.

Kolchin's criteria (See also Khovanskii 2008, Ch. 3, §8). Let the differential Galois group $\mathbf{G}$ of the system (1) be triangular. Then the system is

1. solvable by integrals and algebraic functions if and only if the eigenvalues of all elements of $\mathbf{G}$ are roots of unity;

2. solvable by integrals if and only if the eigenvalues of all elements of $\mathbf{G}$ are equal to unity;

3. solvable by exponentials of integrals and algebraic functions if and only if $\mathbf{G}$ is diagonal;

4. solvable by algebraic functions if and only if $\mathbf{G}$ is diagonal and the eigenvalues of all its elements are roots of unity.

\section{A Local Form of Solutions Near a Singular Point}

In this section, we recall the definitions of regular and irregular singular points of a linear differential system and describe the structure of solutions near a singular point of each type.

A singular point $z=a_{i}$ of the system (1) is said to be regular, if any solution of the system has at most polynomial growth in any sector with the vertex at this point of sufficiently small radius and an opening less than $2 \pi$. Otherwise, the point $z=a_{i}$ is said to be irregular.

A singular point $z=a_{i}$ of the system (1) is said to be Fuchsian, if the coefficient matrix $B(z)$ has a simple pole at this point. Due to Sauvage's theorem, a Fuchsian singular point is always regular (see Hartman 1964, Th. 11.1). However, the coefficient matrix of a system at a regular singular point may in general have a pole of order greater than one. Let us write the Laurent expansion of the coefficient matrix $B$ of the system (1) near its singular point $z=a$ in the form

$$
B(z)=\frac{B_{-r-1}}{(z-a)^{r+1}}+\cdots+\frac{B_{-1}}{z-a}+B_{0}+\cdots, \quad B_{-r-1} \neq 0 .
$$

The number $r$ is called the Poincaré rank of the system (1) at this point (or the Poincaré rank of the singular point $z=a$ ). For example, the Poincaré rank of a Fuchsian singular point is equal to zero.

The system (1) is said to be Fuchsian, if all its singular points are Fuchsian (then it can be written in the form (2)). A system, whose singular points are all regular, will be called regular singular. 


\subsection{A Regular Singular Point}

According to Levelt's theorem Levelt (1961), in a neighbourhood of each regular singular point $a_{i}$ of the system (1), there exists a fundamental matrix of the form

$$
Y_{i}(z)=U_{i}(z)\left(z-a_{i}\right)^{A_{i}}\left(z-a_{i}\right)^{\widetilde{E}_{i}},
$$

where $U_{i}(z)$ is a holomorphic matrix at the point $a_{i}, A_{i}=\operatorname{diag}\left(\varphi_{i}^{1}, \ldots, \varphi_{i}^{p}\right)$ is a diagonal matrix, whose entries $\varphi_{i}^{j}$ are integers organized in a non-increasing sequence, and $\widetilde{E}_{i}=(1 / 2 \pi \mathbf{i}) \ln \widetilde{M}_{i}$ is an upper triangular matrix (the normalized logarithm of the corresponding monodromy matrix $\widetilde{M}_{i}$ ), whose eigenvalues $\rho_{i}^{j}$ satisfy the condition

$$
0 \leqslant \operatorname{Re} \rho_{i}^{j}<1
$$

Such a fundamental matrix is called a Levelt matrix, and one also says that its columns form a Levelt basis in the solution space of the system (in a neighborhood of the regular singular point $a_{i}$ ). The complex numbers $\beta_{i}^{j}=\varphi_{i}^{j}+\rho_{i}^{j}$ are called the (Levelt) exponents of the system at the regular singular point $a_{i}$.

A singular point $a_{i}$ is Fuchsian if and only if the corresponding matrix $U_{i}$ in the decomposition (4) is holomorphically invertible at this point, that is, $\operatorname{det} U_{i}\left(a_{i}\right) \neq 0$. It is not difficult to check that in this case the exponents of the system at the point $a_{i}$ coincide with the eigenvalues of the residue matrix $B_{i}$. In the general case of a regular singularity $a_{i}$, estimates for the order of the function det $U_{i}$ at this point were obtained by Corel (2001) (see also Gontsov 2004):

$$
r_{i} \leqslant \operatorname{ord}_{a_{i}} \operatorname{det} U_{i} \leqslant \frac{p(p-1)}{2} r_{i}
$$

where $r_{i}$ is the Poincaré rank of the regular singular point $a_{i}$. These estimates imply the inequalities for the sum of exponents of a regular singular system over all its singular points, which are called the Fuchs inequalities:

$$
-\frac{p(p-1)}{2} \sum_{i=1}^{n} r_{i} \leqslant \sum_{i=1}^{n} \sum_{j=1}^{p} \beta_{i}^{j} \leqslant-\sum_{i=1}^{n} r_{i}
$$

(the sum of exponents is an integer).

\subsection{An Irregular Singular Point}

Let us now describe the structure of solutions of the system (1) near one of its irregular singular points. We assume that the irregular singularity $z=a$ of Poincaré rank $r$ is non-resonant, that is, the eigenvalues $b_{1}, \ldots, b_{p}$ of the leading term $B_{-r-1}$ of the matrix $B(z)$ in the expansion (3) are pairwise distinct. Let us fix a matrix $T$, which reduces the leading term $B_{-r-1}$ to the diagonal form: 


$$
T^{-1} B_{-r-1} T=\operatorname{diag}\left(b_{1}, \ldots, b_{p}\right)
$$

The system possesses a uniquely determined formal fundamental matrix $\widehat{Y}$ of the form (see Wasow 1965, §§10, 11)

$$
\widehat{Y}(z)=\widehat{F}(z)(z-a)^{\Lambda} e^{Q(z)},
$$

where

(a) $\widehat{F}(z)$ is a matrix formal Taylor series in $z-a$, and $\widehat{F}(a)=T$;

(b) $\Lambda$ is a constant diagonal matrix, whose diagonal entries are called the formal exponents of the system (1) at the irregular singular point $z=a$;

(c) $Q(z)=\operatorname{diag}\left(q_{1}(z), \ldots, q_{p}(z)\right)$ is a diagonal matrix, whose entries $q_{j}(z)$ are polynomials in $(z-a)^{-1}$ of degree $r$ without a constant term,

$$
q_{j}(z)=-\frac{b_{j}}{r}(z-a)^{-r}+o\left((z-a)^{-r}\right) .
$$

For each pair $\left(b_{j}, b_{k}\right)$ of eigenvalues of the matrix $B_{-r-1}$, one has $2 r$ rays starting at the point $a$, which are called Stokes lines of the system (1) at this point:

$$
\begin{gathered}
\left\{z \in \mathbb{C} \mid \operatorname{Re} \frac{b_{j}-b_{k}}{(z-a)^{r}}=0\right\}=\left\{z \in \mathbb{C} \mid \arg (z-a)=\frac{1}{r}\left(\arg \left(b_{j}-b_{k}\right)+\frac{\pi}{2}+\pi m\right),\right. \\
m=0,1, \ldots, 2 r-1\} .
\end{gathered}
$$

These rays are asymptotic to the corresponding curves $\left\{\operatorname{Re}\left(q_{j}-q_{k}\right)=0\right\}$, which divide a neighbourhood of $a$ into domains, in which the function $e^{q_{j}-q_{k}}$ has an exponential growth or decay.

Consider a covering of a punctured neighbourhood of $a$ by $2 r$ congruent sectors

$$
S_{m}=\left\{d+(m-1) \frac{\pi}{r}-\delta<\arg (z-a)<d+m \frac{\pi}{r}+\delta\right\}, \quad m=1, \ldots, 2 r,
$$

with an opening $\frac{\pi}{r}+2 \delta$. The direction $d, 0 \leqslant d<2 \pi$, and small $\delta>0$ can be chosen in such a way that each sector contains exactly one Stokes line for each pair $\left(b_{j}, b_{k}\right)$. Such a covering is often referred to as a good covering by good sectors. According to Sibuya's sectorial normalization theorem (see Ilyashenko and Yakovenko 2008, Th. 21.13, Prop. 21.17), in each good sector $S_{m}$ of a good covering there exists a unique actual fundamental matrix

$$
Y_{m}(z)=F_{m}(z)(z-a)^{\Lambda} e^{Q(z)}
$$

of the system (1), whose factor $F_{m}$ has the asymptotic expansion $\widehat{F}$ in $S_{m}$. In every intersection $S_{m} \cap S_{m+1}$, the fundamental matrices $Y_{m}$ and $Y_{m+1}$ must differ by a constant invertible matrix: 


$$
Y_{m+1}(z)=Y_{m}(z) C_{m}, \quad C_{m} \in \mathrm{GL}(p, \mathbb{C}),
$$

and the logarithmic term $(z-a)^{\Lambda}$ is analytically continued from $S_{1}$ to $S_{2}$, from $S_{2}$ to $S_{3}, \ldots$, from $S_{2 r}$ to $S_{1}$, so that

$$
Y_{1}(z) e^{2 \pi \mathbf{i} \Lambda}=Y_{2 r}(z) C_{2 r} \quad \text { in } S_{2 r} \cap S_{1} .
$$

The matrices $C_{1}, \ldots, C_{2 r}$ are called (Sibuya's) Stokes matrices of the system (1) corresponding to a good covering $\left\{S_{1}, \ldots, S_{2 r}\right\}$ of a punctured neighborhood of a non-resonant irregular singular point $a$. They satisfy the relation

$$
e^{2 \pi \mathbf{i} \Lambda}=M C_{1} \ldots C_{2 r}
$$

where $M$ is the monodromy matrix of $Y_{1}$ at $a$. Indeed, the fundamental matrix $Y_{1}$ can be continued from $S_{1}$ into $S_{2}$ as $Y_{2} C_{1}^{-1}$, since $Y_{1}=Y_{2} C_{1}^{-1}$ in $S_{1} \cap S_{2}$. Further, it is continued from $S_{2}$ into $S_{3}$ as $Y_{3}\left(C_{1} C_{2}\right)^{-1}$, etc. Finally, in $S_{2 r}$ it becomes equal to $Y_{2 r}\left(C_{1} \ldots C_{2 r-1}\right)^{-1}$. Then, according to (8), it comes back into $S_{1}$ as $Y_{1} e^{2 \pi \mathbf{i} \Lambda}\left(C_{1} \ldots C_{2 r}\right)^{-1}$, which implies the relation (9). It is also known that all the eigenvalues of any Stokes matrix are equal to 1 , that is, the Stokes matrices are unipotent (see Ilyashenko and Yakovenko 2008, Prop. 21.19 or Wasow 1965, Th. 15.2).

\section{Local Differential Galois Groups}

Alongside the differential Galois group $\mathbf{G}$ of the system (1), one also defines the local differential Galois groups $\mathbf{G}_{a}$ corresponding to each singular point $z=a$ of the system. Let $\mathcal{M}_{a}=\mathbb{C}(\{z-a\})$ be the field of meromorphic functions at the point $a$. Consider a fundamental matrix $Y_{S}(z)$ of the system (1) on some open sector $S$ with vertex $a$ (its entries are elements of the field of meromorphic functions in $S$ ) and the Picard-Vessiot extension $\mathcal{M}_{a} \subseteq F_{a}=\mathcal{M}_{a}\left\langle Y_{S}\right\rangle$ of $\mathcal{M}_{a}$ generated by all the entries of $Y_{S}$. The corresponding differential Galois group $\mathbf{G}_{a}=\mathrm{Gal}\left(F_{a} / \mathcal{M}_{a}\right)$ is the group of differential automorphisms of the field $F_{a}$ that preserve the elements of the field $\mathcal{M}_{a}$. Like the global differential Galois group $\mathbf{G}$, this group is regarded as an algebraic subgroup of the matrix group $\operatorname{GL}(p, \mathbb{C})$, where this representation depends of course on $a$ and on $Y_{S}$.

We have the following natural inclusion of each $\mathbf{G}_{a}$ into $\mathbf{G}$. Consider a fundamental matrix $Y$ of the system (1) in a neighbourhood $U_{0}$ of a non-singular point $z_{0}$ with respect to which the group $\mathbf{G}$ is determined. The entries of $Y$ are elements of the field of meromorphic functions in $U_{0}$. We analytically continue the matrix $Y$ to the sector $S$ along some path $\gamma$ and obtain a matrix $\widetilde{Y}_{S}$, whose entries are elements of the field $F_{a}$. Then we apply to $\widetilde{Y}_{S}$ some element $\sigma$ of the group $\mathbf{G}_{a}$ and return $\sigma\left(\widetilde{Y}_{S}\right)$ back to $U_{0}$ along the path $\gamma^{-1}$. Since $\mathbb{C}(z) \subset \mathcal{M}_{a}$, the automorphism $\sigma$ preserves rational functions, and thus the transformation $\tau_{\gamma}^{-1} \circ \sigma \circ \tau_{\gamma}$, where $\tau_{\gamma}$ is the operator of an analytic continuation along the path $\gamma$ (we mean that $\tau_{\gamma}$ is an operator from the solution space of (1) in $U_{0}$ to that in $S$ ), is an element of the group $\mathbf{G}$. On the level of matrices, this looks as follows: 


$$
C_{\gamma}^{-1} \mathbf{G}_{a} C_{\gamma} \subset \mathbf{G}
$$

where $C_{\gamma} \in \mathrm{GL}(p, \mathbb{C})$ is a matrix connecting $Y_{S}$ and the analytic continuation $\widetilde{Y}_{S}$ of $Y$ into $S$ along $\gamma$, that is, $\widetilde{Y}_{S}(z)=Y_{S}(z) C_{\gamma}$.

\subsection{Sibuya's Stokes Matrices as Elements of a Local Galois Group}

It was J.-P. Ramis, who described the local differential Galois group $\mathbf{G}_{a}$ in terms of local matrix invariants of the system (1) at the irregular singular point $a$ (among which the Stokes matrices are). He defined (Ramis's) Stokes matrices in a somewhat different way and showed that, in particular, they belong to the matrix group $\mathbf{G}_{a}$ (Ramis 1985; Martinet and Ramis 1989, see also Mitschi 1991; van der Put and Singer 2003, Ch. 8). Further, Ilyashenko and Khovanskii (1990) proved that Sibuya's Stokes matrices defined in the previous section also belong to $\mathbf{G}_{a}$. We will describe their result in more details.

Consider a good covering of a neighbourhood of the singular point $a$ of the system (1) by good sectors $S_{1}, \ldots, S_{2 r}$ and the corresponding fundamental matrices $Y_{1}, \ldots, Y_{2 r}$ described in the previous section. The entries of each $Y_{m}$ are elements of the field of meromorphic functions in the sector $S_{m}$. Let $\mathbf{G}_{a}$ be a matrix group that represents the local differential Galois group of the system (1) at the point $a$ with respect to the fundamental matrix $Y_{1}$ in $S_{1}$. In the intersection $S_{1} \cap S_{2}$, one has $Y_{2}(z)=Y_{1}(z) C_{1}$, where $C_{1}$ is the corresponding Stokes matrix.

Theorem 1 (Ilyashenko and Khovanskii 1990). The matrix $C_{1}$ belongs to the group $\mathbf{G}_{a}$.

In fact, all the Stokes matrices $C_{m}$ are elements of the group $\mathbf{G}_{a}$. Indeed, let $\mathbf{G}_{S_{m}}\left(Y_{m}\right)$ be the matrix group that represents the local differential Galois group of the system (1) at the point $a$ with respect to the fundamental matrix $Y_{m}$ in $S_{m}$ [so that $\mathbf{G}_{S_{1}}\left(Y_{1}\right)=\mathbf{G}_{a}$ ]. In each intersection $S_{m} \cap S_{m+1}$, one has $Y_{m+1}(z)=Y_{m}(z) C_{m}$, and the Stokes matrix $C_{m}$ belongs to $\mathbf{G}_{S_{m}}\left(Y_{m}\right)$ in analogy to Theorem 1 . But all groups $\mathbf{G}_{S_{m}}\left(Y_{m}\right)$ coincide with $\mathbf{G}_{a}$, as can be shown by induction with respect to $m$. Note that an analytic continuation of $Y_{m+1}$ from $S_{m+1}$ into $S_{m}$ is $Y_{m} C_{m}$; hence,

$$
\mathbf{G}_{S_{m+1}}\left(Y_{m+1}\right)=\mathbf{G}_{S_{m}}\left(Y_{m} C_{m}\right)=C_{m}^{-1} \mathbf{G}_{S_{m}}\left(Y_{m}\right) C_{m}=\mathbf{G}_{a}
$$

since $C_{m} \in \mathbf{G}_{S_{m}}\left(Y_{m}\right)$ and $\mathbf{G}_{S_{m}}\left(Y_{m}\right)=\mathbf{G}_{a}$ by the induction hypothesis.

Remark 1 Loday-Richaud (1994) gave an example of a linear differential $2 \times 2$ system that is meromorphically equivalent to a system with a non-resonant irregular singularity $z=0$ of Poincaré rank 1 . The sector $S=\{\operatorname{Re} z>0\}$ does not contain any of the two Stokes lines in that example (thus, $S$ is not "good"); that is why there are two actual fundamental matrices $Y, \widetilde{Y}$ in $S$ with the same asymptotics. Their quotient $C=\left(Y^{-1}\right) \widetilde{Y} \in \mathrm{GL}(2, \mathbb{C})$ is called also a Stokes matrix there (corresponding to the two realizations of one formal fundamental matrix in one sector), and it is shown that $C$ does not belong to the Galois group of the system. 


\subsection{The Exponential Torus}

According to the discussion above, the local differential Galois group $\mathbf{G}_{a}$ (more precisely, its matrix representation with respect to the fundamental solution $Y_{1}=Y_{S_{1}}$ in the good sector $S_{1}$ ) contains the Stokes matrices $C_{1}, \ldots, C_{2 r}$ as well as the formal monodromy matrix $e^{2 \pi \mathbf{i} \Lambda}$ (see the relation (9)). But it also contains a connected subgroup, the exponential torus.

Consider the differential extension $\mathcal{M}_{a} \subset \mathcal{M}_{a}\left\langle e^{q_{1}}, \ldots, e^{q_{p}}\right\rangle$ of $\mathcal{M}_{a}$ generated by the diagonal entries $e^{q_{1}}, \ldots, e^{q_{p}}$ of the exponential factor $e^{Q}$ from the decomposition (6). Let $\mathcal{T}$ denote the differential Galois group of this (Picard-Vessiot) extension, that is, the group of its differential $\mathcal{M}_{a}$-automorphisms. This group may be described in more details as follows.

Let $\left\{p_{1}, \ldots, p_{s}\right\}$ be a basis of the $\mathbb{Z}$-module $\mathbb{Z} q_{1}+\cdots+\mathbb{Z} q_{p}$, so that $\mathcal{M}_{a}\left\langle e^{q_{1}}, \ldots, e^{q_{p}}\right\rangle=\mathcal{M}_{a}\left\langle e^{p_{1}}, \ldots, e^{p_{s}}\right\rangle$. Since the logarithmic derivative of each $e^{p_{i}}$ belongs to $\mathcal{M}_{a}$, for any element $\sigma \in \mathcal{T}$, one has

$$
\sigma\left(e^{p_{1}}\right)=t_{1} e^{p_{1}}, \ldots, \sigma\left(e^{p_{s}}\right)=t_{s} e^{p_{s}}
$$

for some $\left(t_{1}, \ldots, t_{s}\right) \in\left(\mathbb{C}^{*}\right)^{s}$. Conversely, any element of $\left(\mathbb{C}^{*}\right)^{s}$ defines in this way a differential $\mathcal{M}_{a}$-automorphism of $\mathcal{M}_{a}\left\langle e^{p_{1}}, \ldots, e^{p_{s}}\right\rangle$, since there are no differentiallypolynomial relations over $\mathcal{M}_{a}$ between the functions $e^{p_{1}}, \ldots, e^{p_{s}}$. Therefore, $\mathcal{T}$ is a torus of dimension $s$, that is, a linear algebraic group isomorphic to $\left(\mathbb{C}^{*}\right)^{s}$.

To show the inclusion $\mathcal{T} \subset \mathbf{G}_{a}$, first let us extend any $\sigma \in \mathcal{T}$ to a differential $\mathcal{M}_{a^{-}}$ automorphism of the Picard-Vessiot extension $\mathcal{M}_{a} \subset \mathcal{M}_{a}\langle\widehat{Y}\rangle$ generated by all the entries of the matrix $\widehat{Y}$. The latter extension may be viewed as a differential subfield of a larger differential field

$$
\mathcal{L}=\widehat{\mathcal{M}}_{a}\left\langle(z-a)^{\lambda_{1}}, \ldots,(z-a)^{\lambda_{p}}, e^{q_{1}}, \ldots, e^{q_{p}}\right\rangle,
$$

which is an extension of the field $\widehat{\mathcal{M}}_{a}=\mathbb{C}((z-a))$ of formal Laurent series in $z-a$ with a finite principal part generated by the diagonal entries of the matrices $(z-a)^{\Lambda}$, and $e^{Q(z)}$ regarded as formal objects so far. Since

$$
\widehat{\mathcal{M}}_{a}\left\langle(z-a)^{\lambda_{1}}, \ldots,(z-a)^{\lambda_{p}}\right\rangle \cap \widehat{\mathcal{M}}_{a}\left\langle e^{q_{1}}, \ldots, e^{q_{p}}\right\rangle=\widehat{\mathcal{M}}_{a}
$$

one can extend any $\sigma \in \mathcal{T}$ given by (10) onto $\mathcal{L}$ by letting it be the identity map on $\widehat{\mathcal{M}}_{a}\left\langle(z-a)^{\lambda_{1}}, \ldots,(z-a)^{\lambda_{p}}\right\rangle$. Thus, we indeed have a differential $\widehat{\mathcal{M}}_{a}$-automorphism of $\mathcal{L}$ that preserves the Picard-Vessiot extension $\mathcal{M}_{a} \subset \mathcal{M}_{a}\langle\widehat{Y}\rangle$. By restricting every such an automorphism to $\mathcal{M}_{a}\langle\widehat{Y}\rangle$, we identify $\mathcal{T}$ with a subgroup of the differential Galois group of the Picard-Vessiot extension $\mathcal{M}_{a} \subset \mathcal{M}_{a}\langle\widehat{Y}\rangle$. It remains to note that the latter group is isomorphic to $\mathbf{G}_{a}$, because the differential extensions $\mathcal{M}_{a} \subset \mathcal{M}_{a}\langle\widehat{Y}\rangle$ and $\mathcal{M}_{a} \subset \mathcal{M}_{a}\left\langle Y_{1}\right\rangle$ are differentially isomorphic (this is provided by the mapping of $\widehat{Y}$ to $Y_{1}$ via the operation of $r$-summability, which maps here the formal factor $\widehat{F}$ of $\widehat{Y}$ to its sum $F_{1}$ in the sector $S_{1}$ and commutes with differentiation).

Finally, let us write the matrix $T_{\sigma}$ representing an element $\sigma \in \mathcal{T}$ given by (10) with respect to the fundamental solution $Y_{1}$. Let 


$$
\begin{aligned}
q_{1}(z) & =m_{11} p_{1}(z)+\cdots+m_{1 s} p_{s}(z) \\
& \cdots \\
q_{p}(z) & \cdots
\end{aligned}
$$

for some $m_{i j} \in \mathbb{Z}$. Then

$$
\sigma\left(e^{q_{i}}\right)=\sigma\left(e^{m_{i 1} p_{1}} \ldots e^{m_{i s} p_{s}}\right)=t_{1}^{m_{i 1}} e^{m_{i 1} p_{1}} \ldots t_{s}^{m_{i s}} e^{m_{i s} p_{s}}=t_{1}^{m_{i 1}} \ldots t_{s}^{m_{i s}} e^{q_{i}}
$$

hence,

$$
T_{\sigma}=\operatorname{diag}\left(\tau_{1}(t), \ldots, \tau_{p}(t)\right)
$$

where $t=\left(t_{1}, \ldots, t_{s}\right) \in\left(\mathbb{C}^{*}\right)^{s}, \tau_{i}(t)=t_{1}^{m_{i 1}} \ldots t_{s}^{m_{i s}}$. Note that since the functions $q_{1}, \ldots, q_{p}$ are pairwise distinct, the monomials $\tau_{1}(t), \ldots, \tau_{p}(t)$ are pairwise distinct as well.

\section{Linear Differential Systems and Meromorphic Connections on Holomorphic Vector Bundles}

Let us recall some notions concerning holomorphic vector bundles and meromorphic connections in a context of linear differential equations. We mainly follow Bolibruch et al. (2006) or Ilyashenko and Yakovenko (2008), Ch. 3 (see also Gontsov and Poberezhnyi 2008).

In an analytic interpretation, a holomorphic bundle $E$ of rank $p$ over the Riemann sphere is defined by a cocycle $\left\{g_{\alpha \beta}(z)\right\}$, that is, a collection of holomorphic matrix functions corresponding to a covering $\left\{U_{\alpha}\right\}$ of the Riemann sphere:

$$
g_{\alpha \beta}: U_{\alpha} \cap U_{\beta} \longrightarrow \mathrm{GL}(p, \mathbb{C}), \quad U_{\alpha} \cap U_{\beta} \neq \varnothing,
$$

and satisfying the conditions

$$
g_{\alpha \beta}=g_{\beta \alpha}^{-1}, \quad g_{\alpha \beta} g_{\beta \gamma} g_{\gamma \alpha}=I \quad \text { in } U_{\alpha} \cap U_{\beta} \cap U_{\gamma} \neq \varnothing .
$$

Two holomorphically equivalent cocycles $\left\{g_{\alpha \beta}(z)\right\}$ and $\left\{g_{\alpha \beta}^{\prime}(z)\right\}$ define the same bundle. Equivalence of cocycles means that there exists a set $\left\{h_{\alpha}(z)\right\}$ of holomorphic matrix functions $h_{\alpha}: U_{\alpha} \longrightarrow \mathrm{GL}(p, \mathbb{C})$ such that

$$
h_{\alpha}(z) g_{\alpha \beta}(z)=g_{\alpha \beta}^{\prime}(z) h_{\beta}(z)
$$

A section $s$ of the bundle $E$ is determined by a set $\left\{s_{\alpha}(z)\right\}$ of vector functions $s_{\alpha}: U_{\alpha} \longrightarrow \mathbb{C}^{p}$ that satisfy the conditions $s_{\alpha}(z)=g_{\alpha \beta}(z) s_{\beta}(z)$ in non-empty intersections $U_{\alpha} \cap U_{\beta}$.

A meromorphic connection $\nabla$ on the holomorphic vector bundle $E$ is determined by a set $\left\{\omega_{\alpha}\right\}$ of matrix meromorphic differential 1-forms that are defined in the corresponding neighbourhoods $U_{\alpha}$ and satisfy gluing conditions 


$$
\omega_{\alpha}=\left(d g_{\alpha \beta}\right) g_{\alpha \beta}^{-1}+g_{\alpha \beta} \omega_{\beta} g_{\alpha \beta}^{-1}
$$

Under a transition to an equivalent cocycle $\left\{g_{\alpha \beta}^{\prime}\right\}$ connected with the initial one by the relations (11), the 1 -forms $\omega_{\alpha}$ of the connection $\nabla$ are transformed into the corresponding 1 -forms

$$
\omega_{\alpha}^{\prime}=\left(d h_{\alpha}\right) h_{\alpha}^{-1}+h_{\alpha} \omega_{\alpha} h_{\alpha}^{-1}
$$

Conversely, the existence of holomorphic matrix functions $h_{\alpha}: U_{\alpha} \longrightarrow \operatorname{GL}(p, \mathbb{C})$ such that the matrix 1-forms $\omega_{\alpha}$ and $\omega_{\alpha}^{\prime}$ (satisfying the conditions (12) for $g_{\alpha \beta}$ and $g_{\alpha \beta}^{\prime}$ respectively) are connected by the relation (13) in $U_{\alpha}$ implies the equivalence of the cocycles $\left\{g_{\alpha \beta}\right\}$ and $\left\{g_{\alpha \beta}^{\prime}\right\}$ (one may assume that the intersections $U_{\alpha} \cap U_{\beta}$ do not contain singular points of the connection).

Vector functions $s_{\alpha}(z)$ satisfying linear differential equations $d s_{\alpha}=\omega_{\alpha} s_{\alpha}$ in the corresponding $U_{\alpha}$ can be chosen, by virtue of the conditions (12), in such a way that the set $\left\{s_{\alpha}(z)\right\}$ determines a section of the bundle $E$, which is called horizontal with respect to the connection $\nabla$. Thus, horizontal sections of a holomorphic vector bundle with a meromorphic connection are determined by solutions of local linear differential systems. The monodromy of a connection (the monodromy group) characterizes the ramification of horizontal sections under their analytic continuation along loops in $\overline{\mathbb{C}}$ not containing singular points of the connection 1 -forms and is defined similarly to the monodromy group of the system (1). A connection will be called Fuchsian (logarithmic), regular, or irregular, depending on the type of the singular points of its 1 -forms (as singular points of the corresponding linear differential systems).

If a bundle is holomorphically trivial (all matrices of the cocycle can be taken as the identity matrices), then, by virtue of the conditions (12), the matrix 1-forms of a connection coincide on non-empty intersections $U_{\alpha} \cap U_{\beta}$. Hence, horizontal sections of such a bundle are solutions of a global linear differential system defined on the whole Riemann sphere. Conversely, the linear system (1) determines a meromorphic connection on the holomorphically trivial vector bundle of rank $p$ over $\overline{\mathbb{C}}$. Such a bundle has a standard definition by the cocycle that consists of the identity matrices, while the connection is defined by the matrix 1 -form $B(z) d z$ of coefficients of the system. To our purpose, it will be more convenient to use the following equivalent coordinate description [this construction appears already in Bolibruch et al. (2006)].

At first, we consider a covering $\left\{U_{\alpha}\right\}$ of the punctured Riemann sphere $\overline{\mathbb{C}} \backslash\left\{a_{1}, \ldots\right.$, $\left.a_{n}\right\}$ by simply connected neighbourhoods. Then, on the corresponding non-empty intersections $U_{\alpha} \cap U_{\beta}$, one defines the matrix functions of a cocycle, $g_{\alpha \beta}^{\prime}(z) \equiv$ const, which are expressed in terms of the monodromy matrices $M_{1}, \ldots, M_{n}$ of the system (1) via the operations of multiplication and taking the inverse (see Gontsov and Poberezhnyi 2008). In this case, the matrix differential 1 -forms $\omega_{\alpha}^{\prime}$ defining a connection are equal to zero. Further, the covering $\left\{U_{\alpha}\right\}$ is complemented by small neighbourhoods $O_{i}$ of the singular points $a_{i}$ of the system; thus, we obtain the covering of the Riemann sphere $\overline{\mathbb{C}}$. To non-empty intersections $O_{i} \cap U_{\alpha}$, there correspond matrix functions $g_{i \alpha}^{\prime}(z)=Y_{i}(z)$ of the cocycle, where $Y_{i}(z)$ is a germ of a fundamental matrix of the system, whose monodromy matrix at the point $a_{i}$ is equal to $M_{i}$ (so, for analytic 
continuations of the chosen germ to non-empty intersections $O_{i} \cap U_{\alpha} \cap U_{\beta}$, the cocycle relations $g_{i \alpha} g_{\alpha \beta}=g_{i \beta}$ hold). The matrix differential 1-forms $\omega_{i}^{\prime}$ determining the connection in the neighbourhoods $O_{i}$ coincide with the 1-form $B(z) d z$ of coefficients of the system. To prove the holomorphic equivalence of the cocycle $\left\{g_{\alpha \beta}^{\prime}, g_{i \alpha}^{\prime}\right\}$ to the identity cocycle, it is sufficient to check the existence of holomorphic matrix functions

$$
h_{\alpha}: U_{\alpha} \longrightarrow \mathrm{GL}(p, \mathbb{C}), \quad h_{i}: O_{i} \longrightarrow \mathrm{GL}(p, \mathbb{C})
$$

such that

$$
\omega_{\alpha}^{\prime}=\left(d h_{\alpha}\right) h_{\alpha}^{-1}+h_{\alpha} \omega_{\alpha} h_{\alpha}^{-1}, \quad \omega_{i}^{\prime}=\left(d h_{i}\right) h_{i}^{-1}+h_{i} \omega_{i} h_{i}^{-1}
$$

Since we have $\omega_{\alpha}=B(z) d z$ and $\omega_{\alpha}^{\prime}=0$ for all $\alpha$, the first equation in (14) can be rewritten as a linear system

$$
d\left(h_{\alpha}^{-1}\right)=(B(z) d z) h_{\alpha}^{-1},
$$

which has a holomorphic solution $h_{\alpha}^{-1}: U_{\alpha} \longrightarrow \operatorname{GL}(p, \mathbb{C})$, since the 1-form $B(z) d z$ is holomorphic in a simply connected neighbourhood $U_{\alpha}$. The second equation in (14) has a holomorphic solution $h_{i}(z) \equiv I$, since $\omega_{i}=\omega_{i}^{\prime}=B(z) d z$.

One says that the bundle $E$ has a subbundle $E^{\prime} \subset E$ of rank $k<p$ that is stabilized by the connection $\nabla$, if the pair $(E, \nabla)$ admits a coordinate description $\left\{g_{\alpha \beta}\right\},\left\{\omega_{\alpha}\right\}$ of the following block upper triangular form:

$$
g_{\alpha \beta}=\left(\begin{array}{cc}
g_{\alpha \beta}^{1} & * \\
0 & g_{\alpha \beta}^{2}
\end{array}\right), \quad \omega_{\alpha}=\left(\begin{array}{cc}
\omega_{\alpha}^{1} & * \\
0 & \omega_{\alpha}^{2}
\end{array}\right),
$$

where $g_{\alpha \beta}^{1}$ and $\omega_{\alpha}^{1}$ are $k \times k$ blocks (then the cocycle $\left\{g_{\alpha \beta}^{1}\right\}$ defines the subbundle $E^{\prime}$, and the 1 -forms $\omega_{\alpha}^{1}$ define the restriction $\nabla^{\prime}$ of the connection $\nabla$ to the subbundle $E^{\prime}$ ).

Example 1 Consider a system (1), whose monodromy matrices $M_{1}, \ldots, M_{n}$ are of the same block upper triangular form, and the corresponding holomorphically trivial vector bundle $E$ with the meromorphic connection $\nabla$. Suppose that, in a neighbourhood of each singular point $a_{i}$ of the system, there exist a fundamental matrix $Y_{i}(z)$, whose monodromy matrix is $M_{i}$, and a holomorphically invertible matrix $\Gamma_{i}(z)$ such that $\Gamma_{i} Y_{i}$ is a block upper triangular matrix (with the same block structure as the matrix $M_{i}$ ). Let us show that a common invariant subspace of the monodromy matrices gives rise to a vector subbundle $E^{\prime} \subset E$ that is stabilized by the connection $\nabla$.

We use the above coordinate descriptions of the bundle and the connection with the cocycle $\left\{g_{\alpha \beta}^{\prime}, g_{i \alpha}^{\prime}\right\}$ and set $\left\{\omega_{\alpha}^{\prime}, \omega_{i}^{\prime}\right\}$ of matrix 1-forms. The matrices $g_{\alpha \beta}^{\prime}$ are already block upper triangular, since the monodromy matrices $M_{1}, \ldots, M_{n}$ are (and $\omega_{\alpha}^{\prime}=0$ ), while the matrices $g_{i \alpha}^{\prime}=Y_{i}$ can be transformed to such a form, $\Gamma_{i} g_{i \alpha}^{\prime}=\Gamma_{i} Y_{i}$. Thus, changing the matrices $g_{i \alpha}^{\prime}$ to $\Gamma_{i} g_{i \alpha}^{\prime}$ and matrix 1-forms $\omega_{i}^{\prime}$ to $\Gamma_{i} \omega_{i}^{\prime} \Gamma_{i}^{-1}+\left(d \Gamma_{i}\right) \Gamma_{i}^{-1}$, we obtain a holomorphically equivalent coordinate description with the cocycle matrices and connection matrix 1-forms, which have the same block upper triangular form. 
The following auxiliary lemma points to a certain block structure of a linear differential system in the case when the corresponding holomorphically trivial vector bundle with the meromorphic connection has a holomorphically trivial subbundle that is stabilized by the connection.

Lemma 1 If a holomorphically trivial vector bundle $E$ of rank $p$ over $\overline{\mathbb{C}}$ endowed with a meromorphic connection $\nabla$ has a holomorphically trivial subbundle $E^{\prime} \subset E$ of rank $k$ that is stabilized by the connection, then the corresponding linear system (1) is reduced to a block upper triangular form via a constant gauge transformation $\tilde{y}(z)=C y(z), C \in \mathrm{GL}(p, \mathbb{C})$, that is,

$$
C B(z) C^{-1}=\left(\begin{array}{cc}
B^{\prime}(z) & * \\
0 & *
\end{array}\right)
$$

where $B^{\prime}(z)$ is a $k \times k$ block.

Proof Let $\left\{s_{1}, \ldots, s_{p}\right\}$ be a basis of global holomorphic sections of the bundle $E$ (so $s_{1}, \ldots, s_{p}$ are linearly independent at each point $z \in \overline{\mathbb{C}}$ ) such that the 1 -form of the connection $\nabla$ in this basis is the 1 -form $B(z) d z$ of coefficients of the linear system. Consider another basis $\left\{s_{1}^{\prime}, \ldots, s_{p}^{\prime}\right\}$ of global holomorphic sections of the bundle $E$ such that $s_{1}^{\prime}, \ldots, s_{k}^{\prime}$ form a basis of sections of the subbundle $E^{\prime},\left(s_{1}^{\prime}, \ldots, s_{p}^{\prime}\right)=$ $\left(s_{1}, \ldots, s_{p}\right) C^{-1}, C \in \mathrm{GL}(p, \mathbb{C})$.

Now, choose a basis $\left\{h_{1}, \ldots, h_{p}\right\}$ of sections of the bundle $E$ such that $h_{1}, \ldots, h_{p}$ are horizontal with respect to the connection $\nabla$ and $h_{1}, \ldots, h_{k}$ are sections of the subbundle $E^{\prime}$ (this is possible, since $E^{\prime}$ is stabilized by the connection $\nabla$ ). Let $Y(z)$ be a fundamental matrix of the system, whose columns are the coordinates of the sections $h_{1}, \ldots, h_{p}$ in the basis $\left\{s_{1}, \ldots, s_{p}\right\}$. Then

$$
\widetilde{Y}(z)=C Y(z)=\left(\begin{array}{cc}
k \times k & * \\
0 & *
\end{array}\right)
$$

is a block upper triangular matrix, since its columns are the coordinates of the sections $h_{1}, \ldots, h_{p}$ in the basis $\left\{s_{1}^{\prime}, \ldots, s_{p}^{\prime}\right\}$. Consequently, the transformation $\tilde{y}(z)=C y(z)$ reduces the initial system to a block upper triangular form.

The degree deg $E$ (which is an integer) of a holomorphic vector bundle $E$ endowed with a meromorphic connection $\nabla$ may be defined as the sum

$$
\operatorname{deg} E=\sum_{i=1}^{n} \operatorname{res}_{a_{i}} \operatorname{tr} \omega_{i}
$$

of the residues of local differential 1-forms $\operatorname{tr} \omega_{i}$ over all singular points of the connection $\nabla$ (the notation "tr" stays for the trace), where $\omega_{i}$ is the local matrix differential 1 -form of the connection $\nabla$ in a neighbourhood of its singular point $a_{i}$. Later, when calculating the degree of a bundle, we apply the Liouville formula $\operatorname{tr} \omega_{i}=d \ln \operatorname{det} Y_{i}$, where $Y_{i}$ is a fundamental matrix of the local linear differential system $d y=\omega_{i} y$. 


\section{Solvability of a Regular Singular System with Small Exponents}

\subsection{Solvability by Quadratures}

Consider a system (1) with regular singular points $a_{1}, \ldots, a_{n}$ of Poincaré rank $r_{1}, \ldots, r_{n}$ respectively. Here we prove that if the real parts of the exponents of (1) are sufficiently small, then generically solvability of the system (1) by quadratures implies a simple condition on the coefficient matrix $B$.

Theorem 2 Let for some $k \in\{1, \ldots, p-1\}$ the exponents $\beta_{i}^{j}$ of the regular singular system (1) satisfy the conditions

$$
\operatorname{Re} \beta_{i}^{j}>-1 / n k, \quad i=1, \ldots, n, \quad j=1, \ldots, p,
$$

and each difference $\beta_{i}^{j}-\beta_{i}^{l} \notin \mathbb{Q} \backslash \mathbb{Z}$. Then solvability of the system (1) by quadratures implies the existence of a constant matrix $C \in \mathrm{GL}(p, \mathbb{C})$ such that the matrix $C B(z) C^{-1}$ has the following block form:

$$
C B(z) C^{-1}=\left(\begin{array}{cc}
B^{\prime}(z) & * \\
0 & *
\end{array}\right)
$$

where $B^{\prime}(z)$ is an upper triangular $k \times k$ matrix.

Remark 2 Though the inequalities (15) restrict the real parts of the exponents from below, together with the estimates (5) they provide boundedness from above.

Remark 3 The sum of the Poincaré ranks of a regular singular system, whose exponents satisfy the condition (15), is indeed restricted because of the Fuchs inequalities (5), namely, $\sum_{i=1}^{n} r_{i}<p / k$.

The proof of Theorem 2 is based on two auxiliary lemmas.

Lemma 2 Let the exponents $\beta_{i}^{j}$ of the regular singular system (1) satisfy the conditions (15). If the monodromy matrices of this system are upper triangular, then there is a constant matrix $C \in \mathrm{GL}(p, \mathbb{C})$ such that the matrix $C B(z) C^{-1}$ has the form announced in Theorem 2.

Proof We use a geometric interpretation (discussed in the previous section) according to which the regular singular system (1) gives rise to a holomorphically trivial vector bundle $E$ of rank $p$ over the Riemann sphere endowed with a meromorphic connection $\nabla$ with regular singular points $a_{1}, \ldots, a_{n}$.

Since the monodromy matrices $M_{1}, \ldots, M_{n}$ of the system are upper triangular, there exists, as shown in Example 1, a flag $E^{1} \subset E^{2} \subset \cdots \subset E^{p}=E$ of subbundles of rank $1,2, \ldots, p$ respectively that are stabilized by the connection $\nabla$. Indeed, a fundamental matrix $Y$ determining the monodromy matrices $M_{1}, \ldots, M_{n}$ of the system can be represented near each singular point $a_{i}$ in the form

$$
Y(z)=T_{i}(z)\left(z-a_{i}\right)^{E_{i}}, \quad E_{i}=(1 / 2 \pi \mathbf{i}) \ln M_{i},
$$


where $T_{i}$ is a meromorphic matrix at the point $a_{i}$. This matrix can be factored as $T_{i}(z)=V_{i}(z) P_{i}(z)$, with a holomorphically invertible matrix $V_{i}$ at $a_{i}$ and an upper triangular matrix $P_{i}$, which is a polynomial in $\left(z-a_{i}\right)^{ \pm 1}$ (see, for example, Gontsov 2004, Lemma 1). Thus, the matrix $V_{i}^{-1} Y$ is upper triangular.

Let us estimate the degree of each subbundle $E^{j}, j \leqslant k$. For this, we note that, in a neighbourhood of each singular point $a_{i}$, the initial system is transformed via a holomorphically invertible gauge transformation to a system with a fundamental matrix of the form

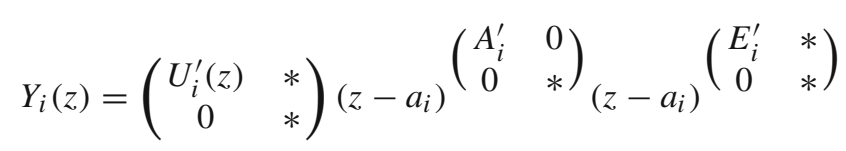

such that the matrix $Y_{i}^{\prime}(z)=U_{i}^{\prime}(z)\left(z-a_{i}\right)^{A_{i}^{\prime}}\left(z-a_{i}\right)^{E_{i}^{\prime}}$ is a Levelt fundamental matrix for a linear system of $j$ equations with the regular singular point $a_{i}$. The matrix 1form of coefficients of this system in a neighbourhood of $a_{i}$ is a local 1-form of the restriction $\nabla^{j}$ of the connection $\nabla$ to the subbundle $E^{j}$, and the exponents $\tilde{\beta}_{i}^{1}, \ldots, \tilde{\beta}_{i}^{j}$ of this system (the eigenvalues of the matrix $A_{i}^{\prime}+E_{i}^{\prime}$ ) form a subset of the exponents of the initial system at $a_{i}$. Therefore,

$$
\operatorname{Re} \tilde{\beta}_{i}^{l}>-1 / n k, \quad l=1, \ldots, j .
$$

The degree of the holomorphically trivial vector bundle $E^{p}$ is equal to zero, and for $j \leqslant k$ one has:

$$
\begin{aligned}
\operatorname{deg} E^{j} & =\sum_{i=1}^{n} \operatorname{res}_{a_{i}} d \ln \operatorname{det} Y_{i}^{\prime}=\sum_{i=1}^{n} \operatorname{ord}_{a_{i}} \operatorname{det} U_{i}^{\prime}+\sum_{i=1}^{n} \operatorname{tr}\left(A_{i}^{\prime}+E_{i}^{\prime}\right) \\
& =\sum_{i=1}^{n} \operatorname{ord}_{a_{i}} \operatorname{det} U_{i}^{\prime}+\sum_{i=1}^{n} \sum_{l=1}^{j} \operatorname{Re} \tilde{\beta}_{i}^{l}>-j / k \geqslant-1
\end{aligned}
$$

Since the degree of a subbundle of a holomorphically trivial vector bundle is nonpositive, one has $\operatorname{deg} E^{j}=0$. Hence, all the subbundles $E^{1} \subset \cdots \subset E^{k}$ are holomorphically trivial (a subbundle of a holomorphically trivial vector bundle is holomorphically trivial, if its degree is equal to zero, see Ilyashenko and Yakovenko 2008, Lemma 19.16). Now the assertion of Lemma 2 follows from Lemma 1.

Definition 1 A matrix will be called $N$-resonant, $N \in \mathbb{N}$, if it has two eigenvalues $\lambda_{1} \neq \lambda_{2}$ such that $\lambda_{1}^{N}=\lambda_{2}^{N}$, that is,

$$
\left|\lambda_{1}\right|=\left|\lambda_{2}\right|, \quad\left|\arg \lambda_{1}-\arg \lambda_{2}\right|=\frac{2 \pi}{N} j, \quad j \in\{1,2, \ldots, N-1\} .
$$

Let a group $\mathbf{M} \subset \mathrm{GL}(p, \mathbb{C})$ be generated by matrices $M_{1}, \ldots, M_{n}$. If these matrices are sufficiently close to the identity (in the Euclid topology), then the existence of a solvable normal subgroup of finite index in $\mathbf{M}$ implies their triangularity, see Theorem 
2.7 in Khovanskii (2008), Ch. 6. According to the remark following this theorem in Khovanskii (2008), the requirement of proximity of the matrices $M_{i}$ to the identity can be weakened as follows.

Lemma 3 There is a number $N=N(p)$ such that if the matrices $M_{1}, \ldots, M_{n}$ are not $N$-resonant, then the existence of a solvable normal subgroup of finite index in $\mathbf{M}$ implies their triangularity.

Proof of Theorem 2 From the theorem assumptions, it follows that the identity component $\mathbf{G}^{0}$ of the differential Galois group $\mathbf{G}$ of the system (1) is solvable; hence, $\mathbf{G}^{0}$ is a solvable normal subgroup of $\mathbf{G}$ of finite index. Then the monodromy group $\mathbf{M}$ of this system also has a solvable normal subgroup of finite index, namely, $\mathbf{M} \cap \mathbf{G}^{0}$.

As follows from the definition of the exponents $\beta_{i}^{j}$ of a linear differential system at a regular singular point $a_{i}$, they are connected with the eigenvalues $\mu_{i}^{j}$ of the monodromy matrix $M_{i}$ by the relation

$$
\mu_{i}^{j}=\exp \left(2 \pi \mathbf{i} \beta_{i}^{j}\right)
$$

Therefore,

$\mu_{i}^{j}=\exp \left(2 \pi \mathbf{i}\left(\operatorname{Re} \beta_{i}^{j}+\mathbf{i} \operatorname{Im} \beta_{i}^{j}\right)\right)=e^{-2 \pi \operatorname{Im} \beta_{i}^{j}}\left(\cos \left(2 \pi \operatorname{Re} \beta_{i}^{j}\right)+\mathbf{i} \sin \left(2 \pi \operatorname{Re} \beta_{i}^{j}\right)\right)$,

and, for any $N \in \mathbb{N}$, the matrices $M_{i}$ are not $N$-resonant by the conditions on the numbers $\beta_{i}^{j}$. Now the assertion of Theorem follows from Lemmas 2 and 3.

As a consequence of Theorem 2, we obtain the following refinement of the Ilyashenko-Khovanskii criterion of solvability by quadratures (mentioned in Introduction) of a Fuchsian system with small residue matrices.

Theorem 3 Let the eigenvalues $\beta_{i}^{j}$ of the residue matrices $B_{i}$ of the Fuchsian system (2) satisfy the conditions

$$
\operatorname{Re} \beta_{i}^{j}>-\frac{1}{n(p-1)}, \quad i=1, \ldots, n, \quad j=1, \ldots, p
$$

and each difference $\beta_{i}^{j}-\beta_{i}^{l} \notin \mathbb{Q} \backslash \mathbb{Z}$. Then solvability of the Fuchsian system (2) by quadratures is equivalent to the simultaneous triangularity of the matrices $B_{i}$.

Proof The necessity of the simultaneous triangularity is a direct consequence of Theorem 2, since the exponents of the Fuchsian system (2) at $a_{i}$ coincide with the eigenvalues of the residue matrix $B_{i}$. The sufficiency follows from a general fact that any linear differential system with an (upper) triangular coefficient matrix is solvable by quadratures (one should solve it beginning with the last equation). 
Remark 4 The inequalities (16) restricting the real parts of the exponents of the Fuchsian system from below also provide their boundedness from above because of the Fuchs relation $\sum_{i=1}^{n} \sum_{j=1}^{p} \beta_{i}^{j}=0$ (see (5)). Namely,

$$
-\frac{1}{n(p-1)}<\operatorname{Re} \beta_{i}^{j}<\frac{n p-1}{n(p-1)} .
$$

In particular, the integer parts $\varphi_{i}^{j}$ (the so-called Levelt valuations) of the numbers $\operatorname{Re} \beta_{i}^{j}$ for such a system must belong to the set $\{-1,0,1\}$.

Remark 5 If each residue matrix $B_{i}$ of the Fuchsian system (2) has only one eigenvalue $\beta_{i}$, then solvability of this system by quadratures is also equivalent to the simultaneous triangularity of the matrices $B_{i}$ (regardless of the values $\operatorname{Re} \beta_{i}$ ). Indeed, in this case each monodromy matrix $M_{i}$ has only one eigenvalue $\mu_{i}=e^{2 \pi \mathbf{i} \beta_{i}}$; hence, it is not $N$-resonant. Therefore, solvability implies the simultaneous triangularity of the monodromy matrices and the existence of a flag $E^{1} \subset E^{2} \subset \cdots \subset E^{p}=E$ of subbundles of the holomorphically trivial vector bundle $E$ that are stabilized by the logarithmic connection $\nabla$ (corresponding to the Fuchsian system). Since the degree $\sum_{i=1}^{n} p \beta_{i}$ of the bundle $E$ is zero, the degree $\sum_{i=1}^{n} j \beta_{i}$ of each subbundle $E^{j}$ is also zero, and all these subbundles are holomorphically trivial.

It is natural to expect that for a general Fuchsian system (with no restrictions on the exponents) solvability by quadratures does not necessarily imply the simultaneous triangularity of the residue matrices. This is illustrated by the following example due to Bolibrukh.

Example 2 (Bolibruch 1994, Prop. 5.1.1). There exist points $a_{1}, a_{2}, a_{3}, a_{4}$ on the Riemann sphere and a Fuchsian system with singularities at these points, whose monodromy matrices are

$$
\begin{aligned}
M_{1}=\left(\begin{array}{rrrrrrr}
1 & 1 & 1 & -1 & 0 & 0 & -1 \\
0 & -1 & -1 & 0 & 0 & 0 & 1 \\
0 & 0 & 1 & 1 & 2 & 2 & 2 \\
0 & 0 & 0 & 1 & 1 & 0 & 1 \\
0 & 0 & 0 & 0 & 1 & 1 & 1 \\
0 & 0 & 0 & 0 & 0 & 1 & 0 \\
0 & 0 & 0 & 0 & 0 & 0 & -1
\end{array}\right), \quad M_{2}=\left(\begin{array}{rrrrrrrr}
1 & 1 & 0 & 1 & 1 & 1 & 0 \\
0 & -1 & 1 & 1 & -1 & 1 & -1 \\
0 & 0 & -1 & -1 & 1 & -1 & 0 \\
0 & 0 & 0 & 1 & 1 & 1 & 0 \\
0 & 0 & 0 & 0 & -1 & -1 & 1 \\
0 & 0 & 0 & 0 & 0 & 1 & 0 \\
0 & 0 & 0 & 0 & 0 & 0 & -1
\end{array}\right), \\
M_{3}=\left(\begin{array}{rrrrrrrr}
1 & 0 & 1 & 0 & -1 & 0 & 0 \\
0 & -1 & -1 & 1 & -1 & 1 & 2 \\
0 & 0 & 1 & 1 & -1 & 1 & 2 \\
0 & 0 & 0 & -1 & 1 & -1 & -2 \\
0 & 0 & 0 & 0 & -1 & 1 & 1 \\
0 & 0 & 0 & 0 & 0 & 1 & 1 \\
0 & 0 & 0 & 0 & 0 & 0 & -1
\end{array}\right), \quad M_{4}=\left(\begin{array}{rrrrrrr}
1 & 0 & 1 & -1 & 1 & 1 & 0 \\
0 & -1 & 1 & -2 & 0 & 0 & 0 \\
0 & 0 & -1 & 1 & 0 & 0 & 0 \\
0 & 0 & 0 & -1 & 1 & 0 & 1 \\
0 & 0 & 0 & 0 & 1 & 1 & 0 \\
0 & 0 & 0 & 0 & 0 & 1 & 1 \\
0 & 0 & 0 & 0 & 0 & 0 & -1
\end{array}\right),
\end{aligned}
$$


whereas the coefficient matrix of this system is not upper triangular. Moreover, this system cannot be transformed in an upper triangular form via neither a constant gauge transformation nor even a meromorphic (rational) one preserving the singular points $a_{1}, a_{2}, a_{3}, a_{4}$ and the pole order (=1) of a coefficient matrix at these points. Thus, the residue matrices of this Fuchsian system are not simultaneously triangular, though the system is solvable by quadratures, since its monodromy group generated by triangular matrices is solvable.

We notice that Theorem 3 does not apply to this example as the exponents of such a system cannot satisfy the conditions (16). Indeed, for any exponent $\beta_{i}^{j}=\varphi_{i}^{j}+\rho_{i}^{j}$, one has

$$
\rho_{i}^{j}=\frac{1}{2 \pi \mathbf{i}} \ln \mu_{i}^{j}, \quad \mu_{i}^{j} \in\{-1,1\}
$$

hence, $\operatorname{Re} \rho_{i}^{j}$ is equal to 0 (for $\mu_{i}^{j}=1$ ) or $1 / 2$ (for $\mu_{i}^{j}=-1$ ). The inequalities (16) imply (for $n=4, p=7$ )

$$
\operatorname{Re} \beta_{i}^{j}>-\frac{1}{24}
$$

hence, $\varphi_{i}^{j}$ is equal to 0 or 1 (see Remark 4). Therefore, the sum of the exponents over all singular points is positive, which contradicts the Fuchs relation.

\subsection{Other Types of Solvability}

At the end of Sect. 2, we gave Kolchin's criteria for a linear differential system to be solvable with respect to different types of solvability formulated in terms of the differential Galois group. For a Fuchsian system with sufficiently small residue matrices, Ilyashenko and Khovanskii (1974) (see also Khovanskii 2008, Ch. 6, §2.3) converted these conditions on the differential Galois group into conditions on the residue matrices. Now this may be formulated in accordance with Theorem 3, while a proof is mainly still the same.

Theorem 4 Under the assumptions of Theorem 3, the Fuchsian system (2) is

1. solvable by integrals and algebraic functions ${ }^{1}$ if and only if all the matrices $B_{i}$ are (simultaneously) triangular and the eigenvalues of each $B_{i}$ are rational numbers differing by an integer;

2. solvable by integrals if and only if all the matrices $B_{i}$ are triangular and their eigenvalues are equal to zero;

3. solvable by exponentials of integrals and algebraic functions ${ }^{2}$ if and only if all the matrices $B_{i}$ are diagonal;

\footnotetext{
${ }^{1}$ Under the assumptions of the theorem, this type of solvability is equivalent to solvability by integrals and radicals.

${ }^{2}$ Under the assumptions of the theorem, this type of solvability is equivalent to solvability by exponentials of integrals.
} 
4. solvable by algebraic functions ${ }^{3}$ if and only if all the matrices $B_{i}$ are diagonal and the eigenvalues of each $B_{i}$ are rational numbers differing by an integer.

Proof We note that each type of solvability implies solvability by quadratures; hence, by Theorem 3 we have a simultaneous triangularity of the residue matrices $B_{i}$. Therefore, the differential Galois group $\mathbf{G}$ of the system is triangular, and we may apply Kolchin's criteria from Sect. 2. As the differential Galois group of a Fuchsian system coincides with the closure of its monodromy group, each condition of the criteria is equivalent to the same condition on the monodromy matrices (each condition determines a Zariski closed set).

So, the eigenvalues of all elements of $\mathbf{G}$ are roots of unity if and only if the eigenvalues of the monodromy matrices are, and, therefore, the eigenvalues of all the residue matrices $B_{i}$ are rational numbers (recall that the eigenvalues of a monodromy matrix $M_{i}$ are $e^{2 \pi \mathbf{i} \beta_{i}^{1}}, \ldots, e^{2 \pi \mathbf{i} \beta_{i}^{p}}$, where $\beta_{i}^{1}, \ldots, \beta_{i}^{p}$ are the eigenvalues of a matrix $B_{i}$ ). Since, according to the assumptions of Theorem $3, \beta_{i}^{j}-\beta_{i}^{l} \notin \mathbb{Q} \backslash \mathbb{Z}$, each difference $\beta_{i}^{j}-\beta_{i}^{l}$ must be an integer.

All the eigenvalues of all elements of $\mathbf{G}$ are equal to 1 if and only if the eigenvalues of the monodromy matrices are; hence, all the eigenvalues of all the residue matrices $B_{i}$ are integers. These cannot be negative in view of (16), but they cannot be positive either in view of the Fuchs relation $\sum_{i=1}^{n} \sum_{j=1}^{p} \beta_{i}^{j}=0$ (if one of them is positive, then there would be another one that is negative).

Finally, $\mathbf{G}$ is diagonal if and only if all the monodromy matrices are. Therefore, a holomorphically trivial vector bundle $E$ over $\overline{\mathbb{C}}$ (with a meromorphic connection $\nabla$ ), which corresponds to the system (2), is a direct sum of line bundles stabilized by $\nabla$. As in the proof of Lemma 2, it follows from (16) that each of these line bundles is holomorphically trivial. Then an evident modification of Lemma 1 proves the reducibility of the Fuchsian system (2) to a diagonal form via a constant gauge transformation.

Notice that, for each type of solvability, the sufficiency of the corresponding condition also may be proved without using differential Galois theory and follows from a method of solving first order non-homogeneous linear differential equations (one begins to solve a Fuchsian system having an upper triangular coefficient matrix with the last equation and uses the method of variation of constants).

It turns out that the class of functions Liouvillian over the field $K=\mathbb{C}(z)$ is closed with respect to the non-algebraic operation of composition. For example, if $f$ is Liouvillian, then $\ln f$ is also Liouvillian, as it is an integral of $f^{\prime} / f$. For an algebraic function $g$, the function $g \circ f$ is Liouvillian: $g$ satisfies an algebraic equation $P(z, g(z))=0$ over $K$, therefore, $g \circ f$ satisfies $P(f(z), g \circ f(z))=0$ and hence is algebraic over $K\langle f\rangle$. However, if we add to the field $K$ some non-Liouvillian function, the situation will change. Consider a field $K\langle F\rangle$, where $F$ is a meromorphic non-Liouvillian function. Then the class of functions Liouvillian over this field is not closed with respect to composition. For example, $\ln F$ is Liouvillian over $K\langle F\rangle$, whereas $F(\ln z)$ is not. Hence, non-solvability of a linear differential equation by quadratures over the field $K\langle F\rangle$ does not guarantee that there is no solution of the form

\footnotetext{
${ }^{3}$ Under the assumptions of the theorem, this type of solvability is equivalent to solvability by radicals.
} 
$F(\ln z)$, for instance. Khovanskii developed a topological version of Galois theory, where composition is equally involved, while the role of the Galois group is played by the monodromy group of a function [for the details see Khovanskii (1995) (Khovanskii 2008, Ch. 5)]. In this theory, one obtains stronger results about non-solvability of linear differential systems. Let $\mathcal{M}$ be the field of meromorphic (in $\mathbb{C}$ ) functions, and let $\mathcal{L}$ be a class of functions that can be obtained from $\mathcal{M}$ by using the standard arithmetic operations, integration, differentiation, as well as composition. If all solutions of the system (1) belong to $\mathcal{L}$, then the monodromy group of the system possesses a solvable normal subgroup of finite index (see Khovanskii 2008, Ch. 6, Cor. 2.13). Systems that cannot be solved in such a way are called strongly non-solvable. Thus, we have the following statement.

Under the assumptions of Theorem 3, the Fuchsian system (2) with non-triangular residue matrices $B_{i}$ is strongly non-solvable.

\section{Solvability of a Non-Resonant Irregular System with Small Formal Exponents}

\subsection{Solvability by Quadratures}

Consider a system (1) with non-resonant irregular singular points $a_{1}, \ldots, a_{n}$ of Poincaré ranks $r_{1}, \ldots, r_{n}$ respectively. If the real parts of the formal exponents of this system are sufficiently small, then the following criterion of solvability by quadratures holds.

Theorem 5 Let at each singular point $a_{i}$ the formal exponents $\lambda_{i}^{j}$ of the irregular system (1) satisfy the conditions

$$
\operatorname{Re} \lambda_{i}^{j}>-\frac{1}{n(p-1)} .
$$

Then this system is solvable by quadratures if and only if there exists a constant matrix $C \in \mathrm{GL}(p, \mathbb{C})$ such that $C B(z) C^{-1}$ is upper triangular.

Proof As in Theorem 3, the sufficiency of the condition does not require a special proof. Let us prove its necessity.

Consider a fundamental matrix $Y$ of the system (1) in a neighbourhood of a nonsingular point $z_{0}$ and the representation of the differential Galois group $\mathbf{G}$ with respect to this matrix (the entries of $Y$ are regarded as elements of the field of meromorphic functions at $z_{0}$ ). For each singular point $a_{i}$, let $Y_{1}^{a_{i}}, \ldots, Y_{2 r_{i}}^{a_{i}}$ be the fundamental matrices of the system defined respectively in the sectors $S_{1}^{a_{i}}, \ldots, S_{2 r_{i}}^{a_{i}}$ of a good covering of a neighbourhood of $a_{i}$ (these sectors were defined at the end of Sect. 3; the upper index $a_{i}$ is used here to emphasize that the involved sectors and matrices correspond to the singular point $a_{i}$ ).

The connection matrix between an analytic continuation $\widetilde{Y}_{S_{1}^{a_{i}}}$ of $Y$ into a neighbourhood of each $a_{i}$ (more precisely, into each first sector $S_{1}^{a_{i}}$ ) and the corresponding $Y_{1}^{a_{i}}$ is denoted by $P_{i}$ : 


$$
\widetilde{Y}_{S_{1}^{a_{i}}}(z)=Y_{1}^{a_{i}}(z) P_{i}, \quad i=1, \ldots, n .
$$

As follows from the explanations of Sect. 4, the matrices

$$
\widetilde{C}_{1}^{a_{i}}=P_{i}^{-1} C_{1}^{a_{i}} P_{i}, \ldots, \widetilde{C}_{2 r_{i}}^{a_{i}}=P_{i}^{-1} C_{2 r_{i}}^{a_{i}} P_{i}, \quad i=1, \ldots, n,
$$

belong to $\mathbf{G}$ (here $C_{1}^{a_{i}}, \ldots, C_{2 r_{i}}^{a_{i}}$ denote the Stokes matrices at the point $a_{i}$, which correspond to the set of fundamental matrices $Y_{1}^{a_{i}}, \ldots, Y_{2 r_{i}}^{a_{i}}$ ). Furthermore, for any set $T_{1}, \ldots, T_{n}$ of diagonal matrices from the exponential tori at the points $a_{1}, \ldots, a_{n}$ respectively, the matrices

$$
\widetilde{T}_{i}=P_{i}^{-1} T_{i} P_{i}, \quad i=1, \ldots, n,
$$

belong to $\mathbf{G}$. Recall that, for each singular point, the corresponding exponential torus consists of all matrices $T(t)=\operatorname{diag}\left(\tau_{1}(t), \ldots, \tau_{p}(t)\right)$, where $t=\left(t_{1}, \ldots, t_{s}\right) \in\left(\mathbb{C}^{*}\right)^{s}$ is an arbitrary parameter, $s$ is the dimension of the torus, and $\tau_{j}$ are pairwise distinct monomials in $t_{1}, \ldots, t_{s}$. Hence, for any $N \in \mathbb{N}$, the $N$-th powers of $\tau_{j}$ are also pairwise distinct monomials. Thus, each matrix $T_{i}$ can be chosen in such a way (by taking a suitable value of $t$ ) that its eigenvalues are pairwise distinct, and that it is not $N$-resonant for any $N \in \mathbb{N}$. This value of $t$ should be taken from the complement $\left(\mathbb{C}^{*}\right)^{S} \backslash \bigcup_{N=1}^{\infty} A_{N}$ of the union $\bigcup_{N=1}^{\infty} A_{N}$ of a countable number of the algebraic subsets

$$
A_{N}=\bigcup_{i \neq j}\left\{t \in\left(\mathbb{C}^{*}\right)^{s} \mid \tau_{i}^{N}(t)=\tau_{j}^{N}(t)\right\} \subset\left(\mathbb{C}^{*}\right)^{s} .
$$

Denote by $\widehat{\mathbf{M}}$ the group generated by the matrices $\widetilde{T}_{i}, \widetilde{C}_{1}^{a_{i}}, \ldots, \widetilde{C}_{2 r_{i}}^{a_{i}}, i=1, \ldots, n$, over all singular points. As follows from the assumptions of the theorem, the group $\mathbf{G}$ possesses the solvable normal subgroup $\mathbf{G}^{0}$ of finite index. Hence, the subgroup $\widehat{\mathbf{M}} \subset$ $\mathbf{G}$ possesses the solvable normal subgroup of finite index, $\widehat{\mathbf{M}} \cap \mathbf{G}^{0}$. For any $N \in \mathbb{N}$, the matrices generating the group $\widehat{\mathbf{M}}$ are not $N$-resonant (the matrices $T_{i}$ are chosen to be non-resonant, whereas for the Stokes matrices this follows from their unipotence). Therefore, according to Lemma 3, the generators of $\widehat{\mathbf{M}}$ are simultaneously reduced to an upper triangular form by conjugating to some invertible matrix $\widetilde{C}$. We may assume that they are already upper triangular (otherwise, we would consider the fundamental matrix $Y \widetilde{C}$ instead of $Y$ ). As follows from Gantmacher (1959), Ch. VIII, §1, the relation

$$
T_{i}=P_{i} \widetilde{T}_{i} P_{i}^{-1}
$$

where $\widetilde{T}_{i}$ is an upper triangular matrix and $T_{i}$ is a diagonal matrix, whose diagonal entries are pairwise distinct, implies that the matrix $P_{i}$ can be written as $P_{i}=D_{i} R_{i}$, where $R_{i}$ is an upper triangular matrix (the conjugation $R_{i} \widetilde{T}_{i} R_{i}^{-1}$ makes all the offdiagonal entries of the matrix $\widetilde{T}_{i}$ zero) and $D_{i}$ is a permutation matrix for $T_{i}$ (that is, the conjugation $D_{i}^{-1} T_{i} D_{i}$ permutes the diagonal entries of the matrix $T_{i}$ ).

In a neighbourhood of each $a_{i}$, we pass from the set of fundamental matrices $Y_{1}^{a_{i}}, \ldots, Y_{2 r_{i}}^{a_{i}}$, which correspond to the sectors $S_{1}^{a_{i}}, \ldots, S_{2 r_{i}}^{a_{i}}$, to the fundamental matrices 


$$
\widetilde{Y}_{m}^{a_{i}}(z)=Y_{m}^{a_{i}}(z) P_{i} \quad\left(\text { in particular, } \widetilde{Y}_{1}^{a_{i}}=Y\right)
$$

connected to each other in the intersections $S_{m}^{a_{i}} \cap S_{m+1}^{a_{i}}$ by the relations

$$
\widetilde{Y}_{m+1}^{a_{i}}(z)=\widetilde{Y}_{m}^{a_{i}}(z) \widetilde{C}_{m}^{a_{i}} .
$$

From the decomposition of $P_{i}$ above and from (7), it follows that the matrices $\widetilde{Y}_{m}^{a_{i}}$ can be written as

$$
\tilde{Y}_{m}^{a_{i}}(z)=F_{m}^{a_{i}}(z)\left(z-a_{i}\right)^{\Lambda_{i}} e^{Q_{i}(z)} D_{i} R_{i}=F_{m}^{a_{i}}(z) D_{i}\left(z-a_{i}\right)^{\Lambda_{i}^{\prime}} e^{Q_{i}^{\prime}(z)} R_{i},
$$

where

$$
\Lambda_{i}^{\prime}=D_{i}^{-1} \Lambda_{i} D_{i}, \quad Q_{i}^{\prime}(z)=D_{i}^{-1} Q_{i}(z) D_{i}
$$

are diagonal matrices obtained from the corresponding matrices $\Lambda_{i}, Q_{i}(z)$ by a permutation of the diagonal entries. Therefore, in the intersections $S_{m}^{a_{i}} \cap S_{m+1}^{a_{i}}$, we have the relations

$$
F_{m+1}^{a_{i}}(z) D_{i}\left(z-a_{i}\right)^{\Lambda_{i}^{\prime}} e^{Q_{i}^{\prime}(z)} R_{i}=F_{m}^{a_{i}}(z) D_{i}\left(z-a_{i}\right)^{\Lambda_{i}^{\prime}} e^{Q_{i}^{\prime}(z)} R_{i} \widetilde{C}_{m}^{a_{i}} .
$$

Thus, in the sectors $S_{1}^{a_{i}}, \ldots, S_{2 r_{i}}^{a_{i}}$, which form a covering of a punctured neighbourhood of $a_{i}$, there are holomorphically invertible matrices $F_{1}^{a_{i}}(z) D_{i}, \ldots, F_{2 r_{i}}^{a_{i}}(z) D_{i}$ such that, in the intersections $S_{m}^{a_{i}} \cap S_{m+1}^{a_{i}}$, their quotients

$$
\left(F_{m}^{a_{i}}(z) D_{i}\right)^{-1} F_{m+1}^{a_{i}}(z) D_{i}=\left(z-a_{i}\right)^{\Lambda_{i}^{\prime}} e^{Q_{i}^{\prime}(z)} R_{i} \widetilde{C}_{m}^{a_{i}} R_{i}^{-1} e^{-Q_{i}^{\prime}(z)}\left(z-a_{i}\right)^{-\Lambda_{i}^{\prime}}
$$

are upper triangular matrices. Since for each $m=1, \ldots, 2 r_{i}$, the matrix $F_{m}^{a_{i}}(z) D_{i}$ has the same asymptotic expansion $\widehat{F}_{i}(z) D_{i}$ in the corresponding sector $S_{m}^{a_{i}}$, there exists a matrix $\Gamma_{i}(z)$ holomorphically invertible at $a_{i}$ such that all the matrices

$$
\widetilde{F}_{m}^{a_{i}}(z)=\Gamma_{i}(z) F_{m}^{a_{i}}(z) D_{i}, \quad m=1, \ldots, 2 r_{i},
$$

are upper triangular (according to Balser et al. 1980, Prop. 3). In particular,

$$
\begin{aligned}
& \Gamma_{i}(z) Y(z)=\Gamma_{i}(z) \widetilde{Y}_{1}^{a_{i}}(z)=\Gamma_{i}(z) F_{1}^{a_{i}}(z) D_{i}\left(z-a_{i}\right)^{\Lambda_{i}^{\prime}} e^{Q_{i}^{\prime}(z)} R_{i} \\
& \quad=\widetilde{F}_{1}^{a_{i}}(z)\left(z-a_{i}\right)^{\Lambda_{i}^{\prime}} e^{Q_{i}^{\prime}(z)} R_{i}
\end{aligned}
$$

is an upper triangular matrix. Hence, for the pair $(E, \nabla)$ consisting of a holomorphically trivial vector bundle $E$ of rank $p$ over $\overline{\mathbb{C}}$ and a meromorphic connection $\nabla$ on it, which corresponds to the system (1), one has a flag $E^{1} \subset E^{2} \subset \cdots \subset E^{p}=E$ of subbundles of ranks $1,2, \ldots, p$ respectively that are stabilized by $\nabla$ (see Example 1).

The final part of the proof, estimates for the degree of each subbundle $E^{j}, j \leqslant p-1$, proceeds as for Lemma 2. Since $E^{j}$ is stabilized by $\nabla$, the initial system, in a neighbourhood of each singular point $a_{i}$, is transformed via a holomorphically invertible gauge transformation into a system with a formal fundamental matrix of the form 


$$
\widehat{Y}_{i}(z)=\left(\begin{array}{cc}
\widehat{F}_{i}^{1}(z) & * \\
0 & *
\end{array}\right)\left(z-a_{i}\right) \stackrel{\left(\begin{array}{cc}
\Lambda_{i}^{1} & 0 \\
0 & \Lambda_{i}^{2}
\end{array}\right)}{{ }^{2}} e^{\left(\begin{array}{cc}
Q_{i}^{1}(z) & 0 \\
0 & Q_{i}^{2}(z)
\end{array}\right)}
$$

such that $\widehat{Y}_{i}^{1}(z)=\widehat{F}_{i}^{1}(z)\left(z-a_{i}\right)^{\Lambda_{i}^{1}} e^{Q_{i}^{1}(z)}$ is a formal fundamental matrix (6) of a local linear differential system of $j$ equations that corresponds to the restriction $\nabla^{j}$ (near $a_{i}$ ) of the connection $\nabla$ to the subbundle $E^{j}$ [existence of the block formal fundamental matrix $\widehat{Y}_{i}$ is proved, for example, in Loday-Richaud (1994), Lemma III.3.8]. Therefore, the formal exponents $\tilde{\lambda}_{i}^{1}, \ldots, \tilde{\lambda}_{i}^{j}$ of this system (the eigenvalues of the diagonal matrix $\Lambda_{i}^{1}$ ) form a subset of the formal exponents of the initial system at $a_{i}$; hence,

$$
\operatorname{Re} \tilde{\lambda}_{i}^{l}>-\frac{1}{n(p-1)}, \quad l=1, \ldots, j .
$$

Thus, for the degree $\operatorname{deg} E^{j}$ one has

$$
\operatorname{deg} E^{j}=\sum_{i=1}^{n} \operatorname{res}_{a_{i}} d \ln \operatorname{det} \widehat{Y}_{i}^{1}=\sum_{i=1}^{n} \operatorname{ord}_{a_{i}} \operatorname{det} \widehat{F}_{i}^{1}+\sum_{i=1}^{n} \operatorname{tr} \Lambda_{i}^{1}+\sum_{i=1}^{n} \operatorname{res}_{a_{i}} d \operatorname{tr} Q_{i}^{1}
$$

Recall that $\operatorname{det} \widehat{F}_{i}^{1}\left(a_{i}\right) \neq 0$ and tr $Q_{i}^{1}$ is a polynomial in $1 /\left(z-a_{i}\right)$; hence,

$$
\operatorname{deg} E^{j}=\sum_{i=1}^{n} \operatorname{tr} \Lambda_{i}^{1}=\sum_{i=1}^{n} \sum_{l=1}^{j} \operatorname{Re} \tilde{\lambda}_{i}^{l}>-\frac{j}{p-1} \geqslant-1 .
$$

This implies (as at the end of the proof of Lemma 2) that all $\operatorname{deg} E^{j}=0$ and that all subbundles $E^{1} \subset \cdots \subset E^{p-1} \subset E$ are holomorphically trivial, which implies the assertion of theorem.

\subsection{Other Types of Solvability}

According to the behaviour of solutions of a linear differential system near its irregular singular point, the system (1) with at least one irregular singular point is not solvable by integrals and algebraic functions. For solvability by exponentials of integrals and algebraic functions the following criterion holds.

Theorem 6 Under the assumptions of Theorem 5, the system (1) is solvable by exponentials of integrals and algebraic functions ${ }^{4}$ if and only if there exists a constant matrix $C \in \mathrm{GL}(p, \mathbb{C})$ such that the matrix $C B(z) C^{-1}$ is diagonal.

Proof The sufficiency of the condition follows immediately from the integration of a system with a diagonal coefficient matrix. To prove the necessity, we note that solvability by exponentials of integrals and algebraic functions implies solvability by quadratures. Hence, by Theorem 5, the coefficient matrix $B$ is reduced to a triangular

\footnotetext{
${ }^{4}$ Under the assumptions of the theorem, this type of solvability is equivalent to solvability by exponentials of integrals.
} 
form via a constant gauge transformation. Therefore, the differential Galois group G of the system (1) is triangular, and we may apply Kolchin's criterion from Sect. 2. According to this criterion, $\mathbf{G}$ is diagonal. Further we proceed as in the proof of Theorem 5 with appropriate simplifications. Namely, since $\widehat{\mathbf{M}} \subset \mathbf{G}$ is diagonal, the Stokes matrices are trivial. Hence, the fundamental matrix $Y(z)$ near each singular point $a_{i}$ has the form

$$
Y(z)=F_{i}(z)\left(z-a_{i}\right)^{\Lambda_{i}^{\prime}} e^{Q_{i}^{\prime}(z)},
$$

where the matrix $F_{i}(z)$ is holomorphically invertible at $a_{i}$. The monodromy matrices of $Y$ are diagonal, the matrix $F_{i}^{-1}(z) Y(z)$ is also diagonal. Therefore, a holomorphically trivial vector bundle $E$ of rank $p$ over $\overline{\mathbb{C}}$ (with a meromorphic connection $\nabla$ on it), which corresponds to the system (1), is a direct sum of line bundles stabilized by $\nabla$ (see Example 1). From the bounds on the formal exponents, it follows again that each of these line bundles is holomorphically trivial. Now a modification of Lemma 1, as mentioned in the proof of Theorem 4, proves the reducibility of the system (1) to a diagonal form via a constant gauge transformation.

As we have mentioned before, a topological version of Galois theory proves that if a Fuchsian system is non-solvable by quadratures, then it is strongly non-solvable. For linear differential equations with irregular singular points this property fails. For example, the equation

$$
y^{\prime \prime}+z y=0
$$

with one (irregular) singular point $z=\infty$ is non-solvable by quadratures (see Kaplansky 1957, Ch. V), but its monodromy is trivial and all solutions are meromorphic in $\mathbb{C}$. Thus, the next natural question is to obtain some sufficient condition for strong nonsolvability of a linear differential system with irregular singular points (formulated in terms of the coefficient matrix rather than monodromy group).

We conclude this subsection by discussing local solvability of a linear differential system by quadratures near its singular point $z=a$. To define this type of solvability, one naturally changes the base field $\mathbb{C}(z)$ of rational functions to the field $\mathcal{M}_{a}$ of meromorphic germs at $a$ and considers Liouvillian extensions of $\mathcal{M}_{a}$. If a Picard-Vessiot extension $\mathcal{M}_{a} \subset F_{a}$ corresponding to the system is contained in some Liouvillian extension of $\mathcal{M}_{a}$, then the system is said to be locally solvable by quadratures near the singular point $z=a$. In analogy to the global case, local solvability by quadratures is equivalent to solvability of the identity component $\mathbf{G}_{a}^{0}$ of the local differential Galois group $\mathbf{G}_{a}$ of the system.

If the singular point $z=a$ is regular, then the system is always locally solvable by quadratures near this point. This follows both from the form (4) of a fundamental matrix

$$
Y(z)=U(z)(z-a)^{A}(z-a)^{\widetilde{E}}
$$

where $U(z)$ is a holomorphic matrix at $a$, and from the fact that the local differential Galois group in this case is the Zariski closure of the local monodromy group, which 
is cyclic, hence solvable. On the other hand, in the case of a non-resonant irregular singular point the following criterion of local solvability by quadratures is obtained along the proof of Theorem 5 .

Theorem 7 A linear differential system is locally solvable by quadratures near its nonresonant irregular singular point if and only if the coefficient matrix is locally reduced to an upper triangular form via a holomorphically invertible gauge transformation at this point.

If a linear differential system has only one singular point (for example, the entries of the coefficient matrix are polynomials), then its local differential Galois group at this point coincides with the global one (see Mitschi 1996, Prop. 1.3). Hence, solvability of such a system by quadratures is equivalent to its local solvability. Thus, we obtain the following consequense of Theorem 7.

Corollary 1 Consider a linear differential system with a polynomial coefficient matrix, whose irregular singular point $z=\infty$ is non-resonant. This system is solvable by quadratures if and only if its coefficient matrix is locally reduced to an upper triangular form via a holomorphically invertible gauge transformation at infinity.

Acknowledgments The authors are very thankful to professor Claude Mitschi for reading the text accurately and giving many improving remarks as well as for kindly providing her (yet unpublished) notes on differential Galois theory from a forthcoming volume of the CIMPA lecture notes (which we essentially used in preparing Sect. 4.2 concerning the exponential torus). We thank professor Askold Khovanskii who has drawn our attention to other types of solvability and has given an advice concerning a presentation of the paper. We also thank the referee for nice and relevant comments and for improving our English. The work is supported by the Russian Foundation for Basic Research (Grant No. 14-01-00346), IUM-Simons Fellowship and Dynasty Foundation.

\section{References}

Balser, W., Jurkat, W.B., Lutz, D.A.: Invariants for reducible systems of meromorphic differential equations. Proc. Edinb. Math. Soc. 23, 163-186 (1980)

Bolibruch, A.A.: The 21st Hilbert problem for linear Fuchsian systems. Proceedings of the Steklov Institute of Mathematics, vol. 206. AMS, Providence, RI (1995)

Bolibruch, A.A., Malek, S., Mitschi, C.: On the generalized Riemann-Hilbert problem with irregular singularities. Expo. Math. 24(3), 235-272 (2006)

Corel, E.: Relations de Fuchs pour les systèmes différentiels réguliers. Bull. S. M. F. 129, 189-210 (2001)

Crespo, T., Hajto, Z: Algebraic groups and differential Galois theory. In: Grad. Stud. Math., vol. 122. AMS (2011)

Gantmacher, F.R.: Theory of Matrices. Chelsea, New York (1959)

Gontsov, R.R.: Refined Fuchs inequalities for systems of linear differential equations. Izv. Math. 68(2), 259-272 (2004)

Gontsov, R.R., Poberezhnyi, V.A.: Various versions of the Riemann-Hilbert problem for linear differential equations. Russ. Math. Surv. 63(4), 603-639 (2008)

Hartman, P.: Ordinary Differential Equations. Wiley, New York (1964)

Ilyashenko, Y.S., Khovanskii, A.G.: Galois theory of differential systems of Fuchsian type with small coefficients (in Russian). Keldysh Inst. Appl. Math. 117, 3-27 (1974)

Ilyashenko, Y.S., Khovanskii, A.G.: Galois groups, Stokes multipliers and Ramis' theorem. Funct. Anal. Appl. 24(4), 286-296 (1990)

Ilyashenko, Y.S., Yakovenko, S.: Lectures on analytic differential equations. In: Grad. Stud. Math., vol. 86. AMS (2008)

Kaplansky, I.: An Introduction to Differential Algebra. Hermann, Paris (1957) 
Khovanskii, A.G.: Topological obstructions to the representability of functions by quadratures. J. Dyn. Control Syst. 1(1), 91-123 (1995)

Khovanskii, A.G.: Topological Galois Theory. Solvability and Non-Solvability of Equations in Finite Terms (in Russian). MCCME, Moscow (2008)

Kolchin, E.R.: Algebraic matrix groups and the Picard-Vessiot theory of homogeneous linear ordinary differential equations. Ann. Math. 49, 1-42 (1948)

Levelt, A.: Hypergeometric functions. Proc. Konikl. Nederl. Acad. Wetensch. Ser. A 64, 361-401 (1961)

Loday-Richaud, M.: Stokes phenomenon, multisummability and differential Galois groups. Ann. Inst. Fourier 44(3), 849-906 (1994)

Martinet, J., Ramis, J.-P.: Théorie de Galois différentielle et resommation. In: Tournier, E. (ed.) Computer Algebra and Differential Equations, pp. 115-214. Academic Press, London (1989)

Mitschi, C.: Differential Galois groups and G-functions. In: Singer, M. (ed.) Computer Algebra and Differential Equations, pp. 149-180. Academic Press, London (1991)

Mitschi, C.: Differential Galois groups of confluent generalized hypergeometric equations: an approach using Stokes multipliers. Pac. J. Math. 176(2), 365-405 (1996)

Ramis, J.-P.: Filtration Gevrey sur le groupe de Picard-Vessiot d'une équation différentielle irrégulière. Informes de Matematica, Preprint IMPA, Serie A-045/85 (1985)

van der Put, M., Singer, M.: Galois Theory of Linear Differential Equations. Springer, Berlin (2003)

Wasow, W.: Asymptotic Expansions for Ordinary Differential Equations. Wiley, New York (1965) 